\title{
Automated Preparation of Core Needle Biopsy Specimens for TEM Imaging
}

Thomas E. Strader ${ }^{*}$ and Benjamin K. August ${ }^{2}$

1. Microscopy Innovations, LLC, Marshfield, WI, USA.

2. University of Wisconsin-Madison School of Medicine and Public Health, Madison, WI, USA.

* Corresponding author: tom.strader@microscopyinnovations.com

Rapid processing of biopsies is critical to both clinicians and patients for timely diagnosis and treatment of disease. Renal pathologists require clear, artefact-free TEM images of multiple glomeruli to make fully informed decisions for evaluation of kidney disease and transplant failure. A critical challenge when preparing core needle biopsies for imaging by TEM is their small size and the need to guard against specimen loss/damage.

Researchers at the University of Wisconsin-Madison and Microscopy Innovations have developed a method suitable for rapid, walk-away processing of core needle biopsies for TEM that is substantially faster than typical 1-2 day protocols. Rapid walk-away TEM preparation is accomplished in 2 hours using mPrep/s capsules and the ASP-1000 Automated Specimen Processor.

A batch of eight perfusion-fixed rodent kidney specimens was collected using a $1 \mathrm{~mm}$ diameter core sampling tool (EMS, Hatfield, PA, USA part \# 69039-10) to approximate an 18-gauge core biopsy (1.02 $\mathrm{mm}$ diameter). The $1 \mathrm{~mm}$ diameter specimens were then trimmed and embedded in low melting point agarose (Sigma Agarose Type I low EEO; CAS Number 9012-36-6) using a reagent trough as a mold. The agar embedded specimens were then cut into several $3 \mathrm{~mm}$ to $5 \mathrm{~mm}$ long specimens (Figures $1 \& 2)$.

For each specimen, an mPrep/s capsule screen was positioned in the mPrep/s Workstation and an embedded biopsy precisely placed vertically on the screen to ensure proper orientation for maximum depth for sectioning (Figure 3). A barcode labeled mPrep/s capsule was then placed over the screen/specimen to securely entrap the specimen for processing and polymerization (Figure 4). After these steps, the capsule with specimen was removed from the Workstation.

The eight mPrep/s capsules containing specimens were then attached to the ASP-1000 for a 2-hour automated processing protocol of buffer, $1 \%$ phosphate buffered $\mathrm{OsO}_{4}$, water, en-bloc staining in 1\% aqueous uranyl acetate, graded ethanols, acetone, and Embed 812 (Figure 6). Following automated processing through $100 \%$ resin, the capsules were removed from the ASP-1000 and polymerized overnight at $60^{\circ} \mathrm{C}$. Barcode labeled mPrep capsules were directly mounted in the microtome chuck for facing and sectioning without post staining.

Electron micrographs were obtained with a Phillips CM120 TEM at $80 \mathrm{keV}$ and recorded to a BioSprint camera. TEM images showing excellent contrast and clearly identifiable glomeruli were generated (Figure5). 

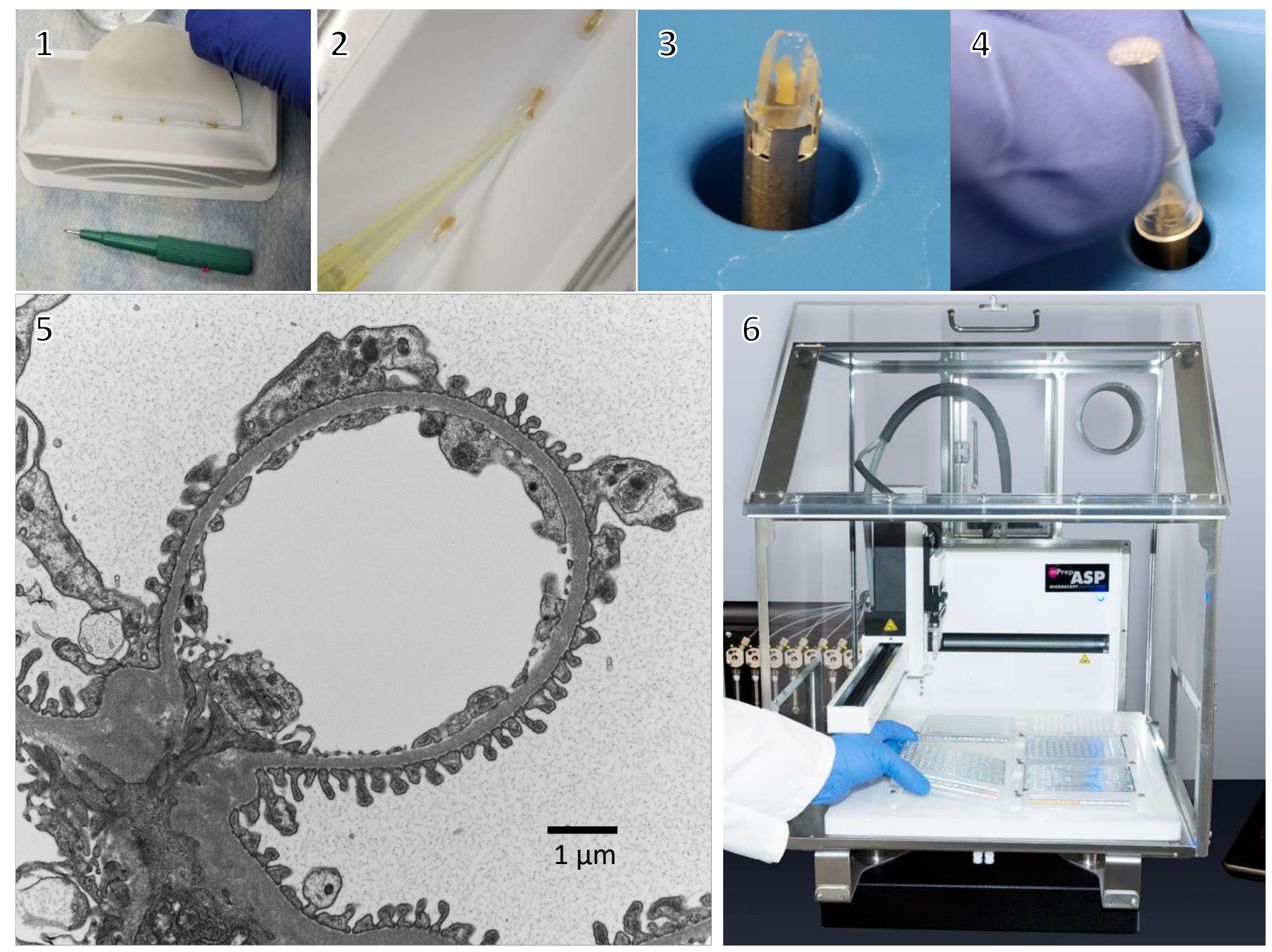

Figure 1. Biopsies are cored with green core sampling tool. Filter paper removes residual buffer.

Figure 2. Warm $50^{\circ} \mathrm{C}$ agar is pipetted on top of biopsy.

Figure 3. The biopsy, in agar, held upright $\mathrm{mPrep} / \mathrm{s}$ Workstation to ensure exact orientation.

Figure 4. $\mathrm{mPrep} / \mathrm{s}$ capsule placed over biopsy to entrap for processing. Barcode label removed to enable viewing the specimen.

Figure 5. TEM image of kidney biopsy tissue

Figure 6. ASP-1000 Automated Specimen Processor 